\title{
Gut microbiota, host genetics and diet interact to affect the risk of developing obesity and the metabolic syndrome
}

New research has shed light on the complex interactions that underlie the development of obesity and the metabolic syndrome. "We were able to identify interactions between host genetics and the microbiota in the intestine that were not previously recognized, and show how these factors interact in creating insulin resistance," explains corresponding author Ronald Kahn (Joslin Diabetes Center and Harvard Medical School, USA).

The researchers imported three inbred strains of mice-C57Bl/6J mice that are

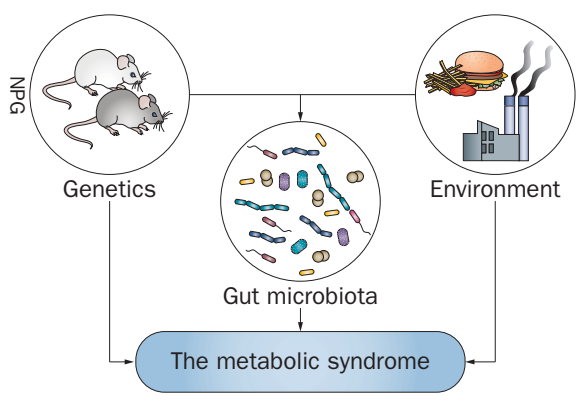

prone to obesity and diabetes mellitus; 129 S1/SvlmJ mice that are resistant to obesity and diabetes mellitus; and 129 S6/SvEvTac mice that are prone to obesity but resistant to diabetes mellitus. They also bred three new lines from these mice in a common environment (B6J/Jos, 129J/Jos and 129T/Jos).

The imported mice developed the expected metabolic phenotypes (such as differing levels of insulin resistance and weight gain) when fed a chow diet or a high-fat diet, which demonstrated the genetic component of the disorders. However, the mice bred at the researchers' laboratory developed different phenotypes to their original strains. This finding suggests that environmental factors interact with host genetics and dietary factors to alter the propensity for developing obesity and the metabolic syndrome.

Kahn and colleagues then used 16S rRNA-based analysis of faecal microbiota to demonstrate that specific bacterial taxa correlated with the different phenotypes, both within and across strains. Some of the phenotypes could also be transferred to germ-free mice using faecal transplantation. Thus, the gut microbiota is likely to be interacting with host genetics and diet to modulate the risk of obesity and the metabolic syndrome.

"Going forward, we are trying to identify both the specific microbes involved in creating insulin resistance and how they do this," says Kahn. "We hope that eventually we will be able to find specific microbiota that promote insulin sensitivity and reduce the metabolic complications of a high-fat diet, such as diabetes mellitus and fatty liver."

Claire Greenhill

Original article Ussar, S. et al. Interactions between gut microbiota, host genetics and diet modulate the predisposition to obesity and metabolic syndrome. Cell Metab. doi:10.1016/i.cmet.2015.07.007 